\title{
AN APPROXIMATE MCKEAN-VLASOV MODEL FOR THE STOCHASTIC FILTERING PROBLEM
}

\author{
DAN CRISAN ${ }^{1}$ AND JiE XIONG ${ }^{2}$
}

\begin{abstract}
The solution of the stochastic filtering problem is approximated using Clark's robust representation approach [1]. The ensuing approximation is shown to coincide with the time marginals of solutions of a certain McKean-Vlasov type process. The result leads to a representation of the solution of the stochastic filtering problem as a limit of empirical distributions of systems of equally weighted particles. A similar representation has been introduced by Del Moral and Miclo in [9] in the context of Feynman-Kac formulae. The representation introduced below differs from the one introduced in [9] as it involves processes with no jumps.
\end{abstract}

\section{INTRODUCTION}

Let $(\Omega, \mathcal{F}, P)$ be a probability space on which we have defined a pair of independent Brownian motions $B=\left(B^{i}\right)_{i=1}^{d}$ and $V=\left(V^{i}\right)_{i=1}^{m}$. Let $X$ be a solution of the stochastic differential equation

$$
d X_{t}=b_{t}\left(X_{t}\right) d t+\sigma_{t}\left(X_{t}\right) d B_{t}
$$

and $W=\left(W^{i}\right)_{i=1}^{m}$ be the stochastic process which satisfies the evolution equation

$$
W_{t}=\int_{0}^{t} \beta_{s}\left(X_{t}\right) d s+V_{t}
$$

Let $\bar{\vartheta}_{t}$ be the conditional distribution of $X_{t}$ given the observation $\sigma$-field $\mathcal{W}_{t}=\sigma\left(W_{s}, s \leq t\right)$. Then $\bar{\vartheta}=$ $\left\{\bar{\vartheta}_{t}, t \geq 0\right\}$ is probability measure valued process satisfying the following non-linear stochastic partial differential equation

$$
\begin{aligned}
\bar{\vartheta}_{t}(\varphi)= & \bar{\vartheta}_{0}(\varphi)+\int_{0}^{t} \bar{\vartheta}_{s}\left(L_{s} \varphi\right) d s \\
& +\sum_{j=1}^{m} \int_{0}^{t}\left(\bar{\vartheta}_{s}\left(\beta_{s}^{j} \varphi\right)-\bar{\vartheta}_{s}\left(\beta_{s}^{j}\right) \bar{\vartheta}_{s}(\varphi)\right)\left(d W_{s}^{j}-\bar{\vartheta}_{s}\left(\beta_{s}^{j}\right) d s\right),
\end{aligned}
$$

where $L$ is the infinitesimal generator associated to $X$ and $\varphi$ is any function in the domain of $L$. Equation (2) is called the Fujisaki-Kallianpur-Kunita or Kushner-Stratonovitch equation (cf. [11], [13]). In general, (2) has

\footnotetext{
1 Department of Mathematics, Imperial College London, London SW7 2BZ, UK.

2 Department of Mathematics, University of Tennessee, Knoxville TN 37996-1300, USA.
} 
no explicit solution, though one can approximate its solution by numerical means. There are a wide variety of methods to do this (see, for example, [3] and the references therein). For example, one can show that $\bar{\vartheta}_{t}$ has the representation

$$
\bar{\vartheta}_{t}=\lim _{n \rightarrow \infty} \frac{\sum_{i=1}^{n} A_{t}\left(X^{i}\right) \delta_{X_{t}^{i}}}{\sum_{i=1}^{n} A_{t}\left(X^{i}\right)}
$$

where $X^{i}, i>0$ are independent copies of $X$ and

$$
A_{t}\left(X^{i}\right)=\exp \left(\sum_{j=1}^{m}\left(\int_{0}^{t} \beta_{s}^{j}\left(X_{s}^{i}\right) d W_{s}^{j}-\frac{1}{2} \int_{0}^{t} \beta_{s}^{j}\left(X_{s}^{i}\right)^{2} d s\right)\right), t \geq 0 .
$$

As demonstrated in [12] representations of the type (3) hold true for a far wider class of stochastic partial differential equations than the one described by (2).

However, the convergence in (3) is very slow. That is because the variance of the weights $A_{t}\left(X^{i}\right), i>$ 0 , increases exponentially fast with time. The effect is that most of weights decrease to 0 with only a few becoming very large. In order to offset this outcome, a wealth of methods have been proposed. In filtering theory, the generic name for such a method is that of a particle filter ([4], [5], [10], etc.). The standard remedy is to introduce an additional procedure that removes particles with small weights and adds additional particles in places where the existing one have large weights. Put differently, one applies at certain times a branching procedure by which, each particle will be replaced by a random number of "offsprings" with a mean proportional with its corresponding weight. This branching procedure is a double edged sword: applying it too often may actually decrease the rate of convergence (cf. [6]).

Here, we suggest a different remedy to the slow convergence of the Monte Carlo method. We will keep the weights of the particles equal without introducing an additional procedure but only by amending the motion of the particles in a way that will take into account the state of the entire system. The proof of the results stated below can be found in [7].

First we need to approximate $\bar{\vartheta}$ by using Clark's robust representation result ([1], see also [2])). For this we introduce $\bar{\vartheta}^{\delta}=\left\{\bar{\vartheta}_{t}^{\delta}, t \geq 0\right\}$ to be the probability measure valued process satisfying the following non-linear partial differential equation

$$
\bar{\vartheta}_{t}^{\delta}(\varphi)=\bar{\vartheta}_{0}(\varphi)+\int_{0}^{t} \bar{\vartheta}_{s}^{\delta}\left(\alpha_{s}^{\delta} \varphi+L_{s} \varphi\right)-\bar{\vartheta}_{s}^{\delta}\left(\alpha_{s}^{\delta}\right) \bar{\vartheta}_{s}^{\delta}(\varphi) d s
$$

where

$$
\alpha_{s}^{\delta}=\sum_{j=1}^{m}\left(\beta_{s}^{j} \frac{W_{(i+1) \delta}^{i}(\omega)-W_{t_{i}}^{i}(\omega)}{\delta}-\frac{1}{2}\left(\beta_{s}^{j}\right)^{2}\right), \quad s \in[i \delta,(i+1) \delta)
$$

Then we can choose $\delta=\delta(\omega, n)$, so that

$$
\left\|\bar{\vartheta}_{t}^{\delta(\omega, n)}-\bar{\vartheta}_{t}\right\|_{\mathcal{M}} \leq \frac{\bar{K}}{\sqrt{n}}
$$

In (7), the norm $\|\cdot\|_{\mathcal{M}}$. is defined as

$$
\|\mu\|_{\mathcal{M}} \overline{\bar{\Delta}} \sum_{k=1}^{\infty} \frac{\left|\mu\left(\varphi_{k}\right)\right|}{2^{k}\left\|\varphi_{k}\right\|}
$$

where $\mu$ is a finite measure and $\left(\varphi_{k}\right)_{k>0}$ are the elements of a convergence determining set $\mathcal{M} \in C_{b}\left(\mathbb{R}^{d}\right)$. 


\section{Main Results}

Let $\mu$ is a probability measure with a strictly positive density $x \rightarrow \mu(x)$ with respect to the Lebesgue measure. Define $(s, x) \longrightarrow \tilde{b}_{s}^{\delta, \mu}(x)$ to be the function

$$
\tilde{b}_{s}^{\delta, \mu}(x) \overline{\bar{\Delta}} b_{s}(x)+\frac{\Lambda_{\mu} \alpha_{s}^{\delta}(x)}{\mu(x)}
$$

where $x \rightarrow \mu(x)$ is the density of $\bar{\vartheta}_{s}^{\delta}$ and $\Lambda_{\mu} f=\left(\Lambda_{\mu}^{j} f\right)_{j=1}^{d}$ is the vector function

$$
\Lambda_{\mu} f(x)=\frac{1}{\omega_{d}} \int_{\mathbb{R}^{d}} \frac{(y-x)(f(y)-\mu(f))}{\|x-y\|^{d}} \mu(d y),
$$

where $f$ is a bounded Borel measurable function and $\mu$ is a probability measure and $\omega_{d}$ is the surface area of the $d$-dimensional sphere $S_{d-1}$. The following are the conditions under which we work:

EU: The coefficients $\alpha_{t}, \beta_{t}^{j}, j=1, \ldots, m, a_{t}^{i j}, i, j=1, \ldots, d, b_{t}^{i}, i=1, \ldots, d$ are bounded (with a bound independent of $t$ ). Further, the functions $a_{t}^{i j}, i, j=1, \ldots, d, b_{t}^{i}, i=1, \ldots, d$ are Lipschitz with a Lipschitz constant independent of $t\left(a=\sigma_{s} \sigma_{s}^{\top}\right)$. The operator $L$ is uniformly elliptic.

PD: $\vartheta_{0}$ has finite second moment, is absolutely continuous with respect to the Lebesgue measure and its density is strictly positive everywhere.

ES: The function $x \longrightarrow \tilde{b}_{s}^{\delta, \bar{\vartheta}_{s}^{\delta}}(x)$ is globally Lipschitz uniformly on $[0, t]$ for any $t \geq 0$.

The second step is to prove that $\bar{\vartheta}_{t}^{\delta}$ can be represented as the one-dimensional time marginals of a non-linear diffusion satisfying a certain McKean-Vlasov equation. We have the following:

Theorem 2.1. Let $(\tilde{\Omega}, \tilde{\mathcal{F}}, \tilde{P})$ be a probability space on which there exists a process $X=\left(X_{t}^{\delta}\right)_{t \geq 0}$ which satisfies equation

$$
X_{t}^{\delta}=X_{0}^{\delta}+\int_{0}^{t} \tilde{b}_{s}^{\delta, \tilde{\vartheta}_{s}^{\delta}}\left(X_{s}^{\delta}\right) d s+\int_{0}^{t} \sigma_{s}\left(X_{s}^{\delta}\right) d B_{s}
$$

where $B=\left(B_{t}\right)_{t>0}$ is a d-dimensional Brownian motion and $X_{0}^{\delta}$ has distribution $\bar{\vartheta}_{0}$. In $(10)$, $\tilde{\vartheta}_{s}^{\delta}$ is the distribution of $X_{s}^{\delta}$. Then, under conditions $\mathbf{E U}+\mathbf{P D}, \tilde{\vartheta}_{t}^{\delta}$ and $\bar{\vartheta}_{t}^{\delta}$ coincide for all $t \geq 0$.

Theorem 2.2. Under the conditions $\mathbf{E U}+\mathbf{P D}+\mathbf{E S}$, equation (10) has a unique solution.

We have the following convergence result.

Theorem 2.3. For a suitable choise of the parameter $\delta_{n}$, there exists a constant $k$ independent of $n$ suchthat

$$
\tilde{E}\left[\left\|\bar{\vartheta}_{t}-\bar{\vartheta}_{t}^{\delta_{n}}\right\|_{\mathcal{M}}^{4}\right] \leq \frac{k}{n^{2}}
$$

In particular, $\bar{\vartheta}_{t}^{\delta_{n}}$ converges to $\bar{\vartheta}_{t}, \tilde{P}$-almost surely and

$$
\bar{\vartheta}_{t}=\lim _{n \rightarrow \infty} \frac{1}{n} \sum_{i=1}^{n} \delta_{X_{t}^{i, \delta_{n}}}
$$

where $X^{i, \delta_{n}}, i \geq 0$ are independent realizations of the non-linear diffusion $X^{\delta}$ as defined by (10). Hence $\bar{\vartheta}_{t}$ has an asymptotic representation involving particles with equal weights. 
We note that if the density of $\vartheta_{s}^{\delta}$ has the form $\vartheta_{s}^{\delta}(x)=e^{-F_{s}^{\delta}(x)}, x \in \mathbb{R}$, where $F_{s}^{\delta}$ is a differentiable convex function, then condition ES is satisfied. The construction also works with minimal changes when (the initial Markov process) $\xi$ is a reflecting boundary diffusion. In this case, the analysis simplifies considerably if the domain is compact. In this case, condition ES is replaced by the assumption that $X$ has a density which is bounded away from 0 .

The asymptotic representations (11) is not, in itself, a direct numerical methods for solving the SPDE (2). For this, one would have to obtain numerical approximation for the non-linear diffusions $\left\{X^{i, \delta}\right\}_{i=1}^{n}$. In other words, we need to solve numerically the McKean-Vlasov equation (10). An additional approximating procedure is needed for this. See [8] for details.

\section{REFERENCES}

[1] J. M. C. Clark, The design of robust approximations to the stochastic differential equations of nonlinear filtering. Communication systems and random process theory (Proc. 2nd NATO Advanced Study Inst., Darlington, 1977), pp. 721-734. NATO Advanced Study Inst. Ser., Ser. E: Appl. Sci., No. 25, Sijthoff \& Noordhoff, Alphen aan den Rijn, 1978.

[2] J. M. C. Clark, D. CRISAN, On a robust version of the integral representation formula of nonlinear filtering, Probability Theory and Related Fields, 2005, Vol 133, No 1 pp 43-56.

[3] D. CRISAN, Numerical methods for solving the stochastic filtering problem, Numerical methods and stochastics (Toronto, ON, 1999), 1-20, Fields Inst. Commun., 34, Amer. Math. Soc., Providence, RI, 2002.

[4] D. CRisan, T. Lyons, A particle approximation of the solution of the Kushner-Stratonovitch equation Probab. Theory Related Fields 115 no. 4, pp 549-578, 1999.

[5] D. Crisan, P. Del Moral T. Lyons, Interacting particle systems approximations of the Kushner-Stratonovitch equation. Adv. in Appl. Probab. 31, no. 3, pp 819-838, 1999.

[6] D. CRISAn, Exact rates of convergence for a branching particle approximation to the solution of the Zakai equation Ann. Probab. 31, no. 2, 693-718, 2003.

[7] D. Crisan, J. Xiong, Approximate McKean-Vlasov Representations for a class of SPDEs, http://arxiv.org/find/math/1/au:+Crisan_D/0/1/0/all/0/1.

[8] D. Crisan, J. Xiong, Numerical Solutions for a Class of SPDEs over bounded domains, these Proceedings.

[9] P. Del Moral, L. Miclo, A Moran particle system approximation of Feynman-Kac formulae. Stochastic Process. Appl. 86 (2000), no. 2, 193-216.

[10] P. Del Moral, L. Miclo, Branching and interacting particle systems approximations of Feynman-Kac formulae with applications to non-linear filtering. Séminaire de Probabilités, XXXIV, pp 1-145, Lecture Notes in Math., 1729, Springer, Berlin, 2000.

[11] M. Fujisaki, G. Kallianpur, H. Kunita, Stochastic differential equations for the non linear filtering problem. Osaka J. Math. 9, pp 19-40, 1972 .

[12] T. G. Kurtz, J. Xiong, Particle Representation for a Class of Nonlinear SPDE's, Stochastic Process. Appl. 83, no. 1, pp 103-126, 1999

[13] H. J. Kushner, Dynamical equations for optimal nonlinear filtering, J. Differential Equations 3, pp 179-190, 1967. 